\title{
Research on Life Prediction of Fan Spindle Bearing Based on Gated Recurrent Unit Neural Network
}

\author{
Ma Jianeng, ${ }^{1, *}$ \\ ${ }^{1}$ Shanghai DianJi University, Shanghai, 201306, China
}

\begin{abstract}
In order to solve the problems of redundancy calculation and inefficiency of traditional machine learning algorithm in dealing with large amount of historical data of fan, a new predictive algorithm based on gated recurrent unit (GRU) is proposed to predict the remaining service life of fan spindle bearing. Firstly, the vibration history data of the main shaft bearing of the fan is analyzed to find out the relationship between the characteristic value and the remaining life, and the characteristic parameters which can reflect the remaining life are selected; Then, GRU is used to build the remaining service life prediction model of spindle bearing, and the main parameters of the model are adjusted to improve the prediction accuracy of the model. Compared with long short term (LSTM) algorithm, GRU is an effective tool to deal with a large number of data sets.
\end{abstract}

\section{Introduction}

With the strong growth of global energy consumption, the contradiction between supply and demand is worsening, and the rapid economic development makes the global demand for energy unprecedented. In order to solve the energy crisis and alleviate the impact of fossil energy, renewable energy has been widely concerned with its unique advantages. Among them, wind energy, as the most mature technology, the most large-scale development conditions and commercial development prospects of renewable energy, has been widely valued and vigorously developed by all countries.

In the drive system of wind turbine, as the key component of the drive system of wind turbine, the spindle bearing bears the weight of large components such as blades, hubs and pitch system of wind turbine and bears the task of transferring the torque generated by the rotation of blades. Therefore, the spindle bearing plays a vital role. Once the main shaft bearing breaks down, it is difficult to repair. And it will consume a lot of maintenance time and cost, which will seriously affect the efficiency of the whole wind farm. Therefore, it is necessary to real-time condition monitoring of wind power bearings, performance degradation process analysis and residual life prediction research to provide technical support for operation and maintenance of wind power units, so as to improve the reliability and availability of the whole unit.

At present, the commonly used life prediction methods mainly include two methods, one is based on failure physical model and the other is based on data-driven method. The former does not need to count a lot of historical data, and the latter is based on a lot of historical data to establish the functional relationship between the observed amount and the prediction model, and then to forecast the remaining life. In recent years, many scholars at home and abroad have carried out research on bearing life prediction and achieved corresponding research results. In [1], the method of self-organizing neural network and back propagation neural network is used to predict the remaining life of bearing. In [2], according to the vibration signal of the gearbox, the relationship between the degradation state and the eigenvalue of the gearbox is established, and the residual life of the gearbox is accurately estimated by the particle filter algorithm. In [3], the remaining life of lithium-ion battery is predicted by using neural network based on quantum particle swarm optimization.

However, the above research based on traditional machine learning algorithm is only applicable to wired data sample space, and it is inefficient to deal with large sample data sets, so it is difficult to meet the actual needs of engineering. Therefore, this paper proposes a Gru based prediction method for the remaining service life of spindle bearing. Firstly, the original vibration signal is extracted as the input of the prediction model. A part of the samples are input into the GRU prediction model as training set, and the model is trained in batches, and the network parameters are adjusted. Finally, the test set is used to test the built model, and compared with the LSTM network model, the effectiveness and efficiency of the experiment are verified.

\section{The gated recurrent unit neural network}

In recent years, with the continuous development of deep learning technology, some deep learning models are gradually applied to the research of time series data. Deep learning model is a kind of deep neural network model with multiple nonlinear mapping levels, which can 
abstract and extract features from the input signal layer by layer, and dig out deeper potential rules.

Among many deep learning models, recurrent neural network (RNN) is a kind of neural network which is suitable for processing time series data. By introducing feedback mechanism, the time dependence of the series is obtained, so that the network model has memory function.

The LSTM neural network not only inherits the advantages of RNN processing time series data, but also solves the disadvantages of RNN gradient disappearance and gradient explosion. However, due to the complexity of LSTM network structure, it takes a long time in network training and prediction. By simplifying and improving the structure of LSTM, the variant GRU neural network of LSTM is obtained. GRU and LSTM have similar prediction accuracy, but GRU has simpler structure, fewer parameters and better convergence. Therefore, GRU is selected as the remaining service life prediction algorithm in this paper.

\subsection{Basic principles of GRU prediction model}

The structure of GRU neural network is shown in Figure 1. There are also input layer, hidden layer and output layer in GRU neural network, and the output layer and hidden layer are the same. The input of GRU module A includes the input $x_{t}$ of the current time and the hidden layer state $h_{t-1}$ of the previous time. The content of $h_{t-1}$ and $o_{t-1}$ output layer is the same. The loop structure makes the hidden layers of GRU modules interconnected, so that the timing information can be transferred from the previous time to the next time.

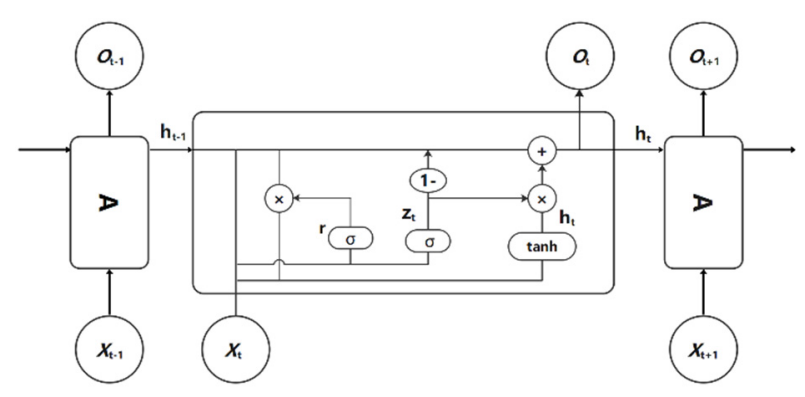

Fig.1. Unfolded structure of GRU network

Two gate functions, update gate $z_{t}$ and reset gate $r_{t}$, are introduced into the GRU cell structure. Among them, the update gate is simplified and integrated by the input gate and forgetting gate of LSTM, and the original cell state and hidden state are mixed and changed. The process of updating the status information of GRU unit is as follows: the output $h_{t-1}$ of the hidden layer at the previous time is transmitted to the current time through the update gate $z_{t}$, and the influence degree of the hidden layer state $h_{t-1}$ at the previous time on the current layer is determined by controlling the value of the update gate; the current input $h_{t-1}$ and $z_{t}$ are written into the current candidate hidden state $\tilde{\mathrm{h}}_{\mathrm{t}}$, in which the writing degree of $h_{t-1}$ is controlled by the reset gate $r_{t}$, and the value of the reset gate $r_{t}$ is reset. The larger it is, the more information is written; The current hidden state depends on $h_{t-1}$ and $\tilde{\mathrm{h}}_{\mathrm{t}}$. Thus, the time series information can be filtered, superimposed, calculated and updated, and the efficiency of the model can be improved. The relationships among the variables are as follows:

$$
\begin{aligned}
& z_{t}=\sigma\left(W_{z} \cdot\left[h_{t-1}, x_{t}\right]\right) \\
& r t=\sigma\left(W_{r} \cdot\left[h_{t-1}, x_{t}\right]\right) \\
& \tilde{h}_{t}=\tanh \left(W \cdot\left[r_{t}^{*} h_{t-1}, x_{t}\right]\right) \\
& h_{t}=(1-z t) * h_{t-1}+z_{t} * \tilde{h}_{t}
\end{aligned}
$$

Where: $W_{z}, W_{r}$ and $W$ are updating the door weight, resetting the door weight and weight coefficient respectively; $\sigma$ is the ReLU activation function with the value range of $[0,1] ; *$ is the inner product of the vector.

\section{Methods and steps of the experiment}

To accurately predict the remaining service life of the bearing, we need to solve the following two key problems: first, how to quickly and accurately identify the degradation state of the bearing according to the historical vibration data, and determine the degradation time point of the bearing. Secondly, how to select the characteristic parameters which can reflect the bearing degradation trend to characterize the bearing degradation process. Based on the above two problems, this paper proposes a prediction method of rolling bearing residual service life based on gated recurrent unit neural network, and the flow chart of the method is shown in Figure 2. The specific steps are as follows:

\subsection{Data processing}

The whole life cycle of bearing has three stages: running in period, validity period and decline period. A total of 984 groups of data were used in this project, and 20480 points were sampled in each group. 550 points after each group of data are selected to analyze the whole life degradation process of bearing. According to the magnitude of bearing vibration amplitude, the time point of bearing entering the decline period is determined, and the data in the decline period is re selected to fit and analyze the bearing degradation trend in the decline period, and extract the characteristic information of bearing degradation. For the first and middle stage of the bearing, the data is in a relatively stable state. This process can be regarded as the stable operation stage of the bearing, so it is not analyzed in the experiment. 


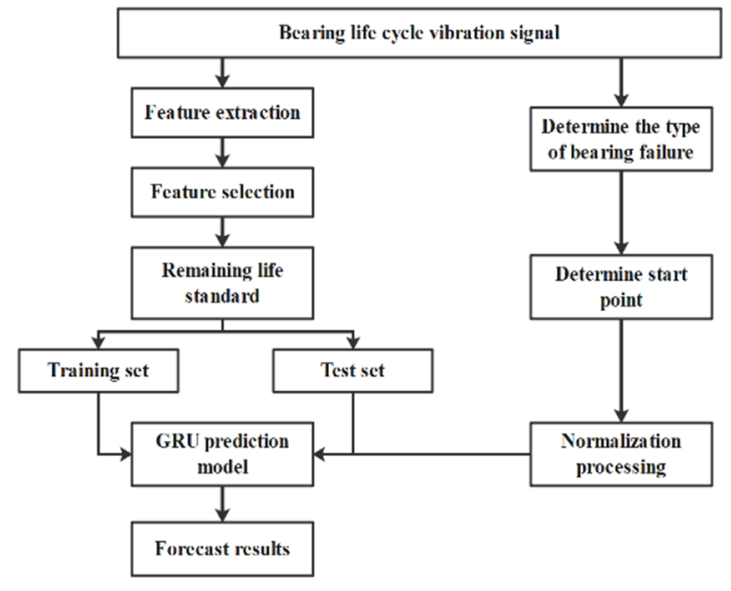

Fig.2. Method flow chart

\subsection{Feature parameter extraction}

In signal feature extraction, time domain statistics is the most basic signal feature extraction method, which has the advantages of large amount of information, strong intuition, and can directly reflect the characteristics of vibration signal. Through the time-domain distribution characteristics of discrete sampling points, the variation law can be described intuitively, which is widely used in the field of bearing fault feature extraction. The common time-domain characteristic indexes include dimensional and non dimensional indexes, such as variance, extreme value, square root amplitude, peak value, etc. However, single selection of a time-domain parameter for feature extraction has some disadvantages, such as unclear expression of feature information and indetail timedomain index. In order to effectively characterize the running condition of bearings, this paper considers the combination of several time-domain features for trend prediction. Therefore, this paper selects several typical time-domain characteristic parameters including variance, root mean square value, effective value, maximum value, extreme value, root mean square amplitude, kurtosis, skewness, peak value, margin and so on for feature extraction, so as to realize the effective generalization of bearing life cycle under multiple time-domain characteristic sets.

Ten characteristic parameters which can reflect the characteristics of bearing degradation are selected to form a 10 dimensional feature vector. The construction of eigenvector set can effectively cover the variation of multiple performance parameters of bearing vibration signal. The construction of high-dimensional feature vector set satisfies the information breadth, and then uses normalization to reduce the dimension of feature vector to avoid the redundancy and conflict between feature vectors.

\section{Bearing life prediction experiment}

The bearing life cycle test data used in this experiment are provided by the intelligent system maintenance center of University of Cincinnati[4]. The schematic diagram of life cycle test bench is shown in Figure 3.

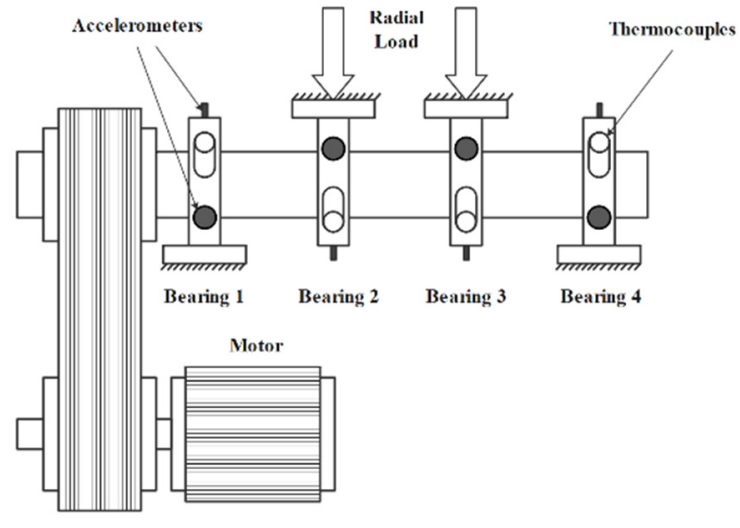

Fig.3. Structure of test bed

There are four double row roller bearings in the main shaft of the test-bed, and the bearing model is Rexnord ZA-2115. The two ends of the bearing are fixed and driven by the motor through the belt. The speed is $2000 \mathrm{r} / \mathrm{min}$. A radial load of $26.67 \mathrm{KN}$ is applied to the bearing, and the vibration signals from the bearing are collected by two acceleration sensors. The data is collected every 10 minutes, and the sampling frequency is $20 \mathrm{kHz}$. During the experiment, each bearing was forced to be lubricated, and the temperature of the outer ring of the bearing was measured by thermocouples equipped on four bearing seats to realize real-time monitoring. The fault degree of gyroscope is judged by the magnetic plug in the oil return pipe of the lubrication system. The magnetic plug collects the metal abrasive particles in the lubricating oil as the fault measurement standard. When the number of metal particles reaches a certain level, it indicates that the bearing has serious fault, so the experiment ends. In this paper, the second set of data is used to test the bearing 1 . After 164 hours of operation, the outer ring of the bearing fails seriously.

Take the life data of bearing 1 and bearing 2 as an example, and the vibration signal is shown in Figure 4. It can be seen from the figure that bearing 2 has been in a stable operation state during the operation. The vibration amplitude of bearing 1 starts with steady fluctuation, then increases, and then increases sharply in the last stage. The whole degradation trend is in line with the three degradation stages of the bearing. Because the sampling interval is 10 minutes, the running time of bearing can be obtained by sampling points, and the starting time of bearing degradation can be determined according to the amplitude of vibration. According to the calculation, the bearing 1 has slight wear after about 117 hours of operation. After 150 hours of operation, the vibration trend of bearing changes obviously, and the bearing has serious failure.

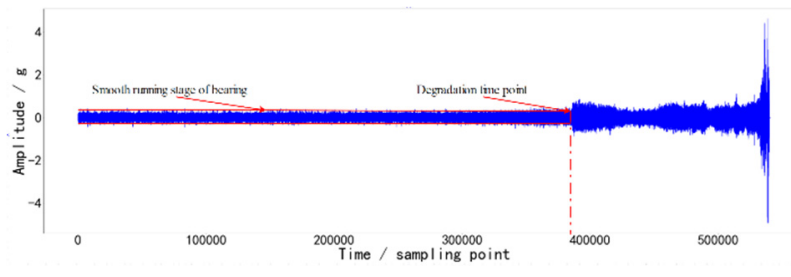




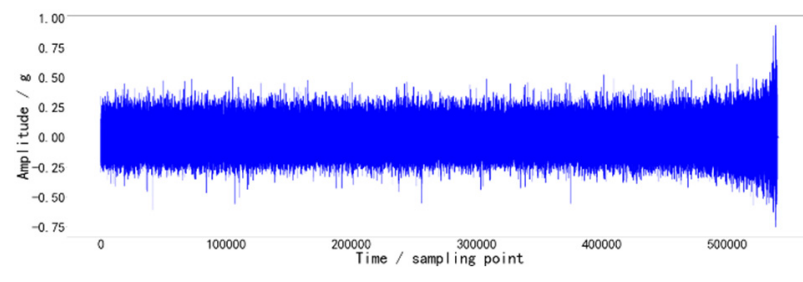

Fig.4. Life cycle vibration signals of bearing 1 and bearing 2

A good characteristic index can effectively judge the bearing degradation, that is, it needs to be sensitive to the initial degradation state of the bearing, and its change trend can be relatively consistent with the whole process of the bearing degradation state, but some of the above conditions can not be met, such as the average value and kurtosis, as shown in Figure 5.

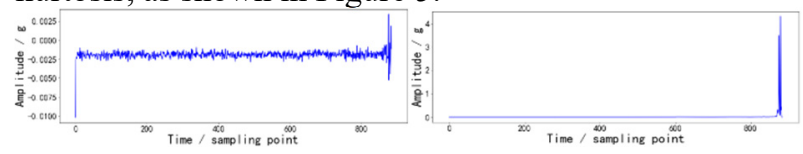

Fig.5. Trend of mean and kurtosis

As can be seen from Figure 5, the variation trend of average value and kurtosis is not consistent with the bearing degradation process, and this inconsistency makes it impossible to judge the degree of bearing performance degradation according to its variation trend. The trend change chart of standard deviation, variance, skewness and peak value is shown in Figure 6.

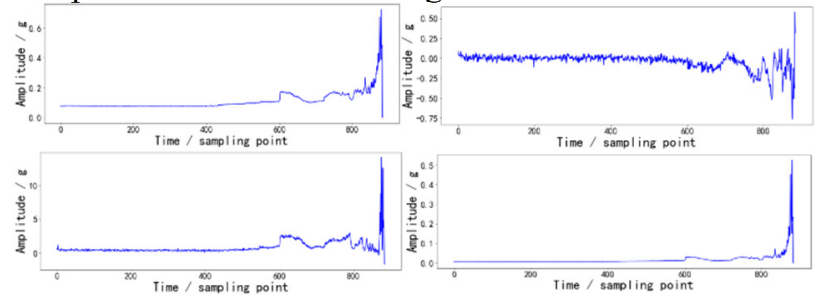

Fig.6. Trend chart of standard deviation, variance, skewness and peak value

It can be seen from Figure 6 that the peak value and root mean square value have good stability. When the rolling bearing is worn, it can well show the general trend of bearing degradation state, which is more conducive to improving the accuracy of model evaluation. According to the same method, 10 effective feature parameters were selected from 16 feature parameters.

In order to judge when the bearing reaches the expected life, 550 groups of experimental data are divided according to the ratio of 7 to 3 . The first 285 groups are used as the training set. The model is trained through the training set, and then the remaining 165 groups of test sets are input into the model. Figure 7 shows the prediction result of bearing 1, in which the abscissa is the number of samples and the ordinate is the degradation percentage of the corresponding samples, that is, the normalized life multiplied by 100 , indicating the percentage of the sample from the aging point. The red line is the actual remaining life of the bearing, the blue line is the remaining life predicted by GRU model, and the green line is the remaining service life predicted by LSTM model. It can be seen from Figure 7 that the prediction errors of the two methods are almost the same, and the prediction trend is almost the same.

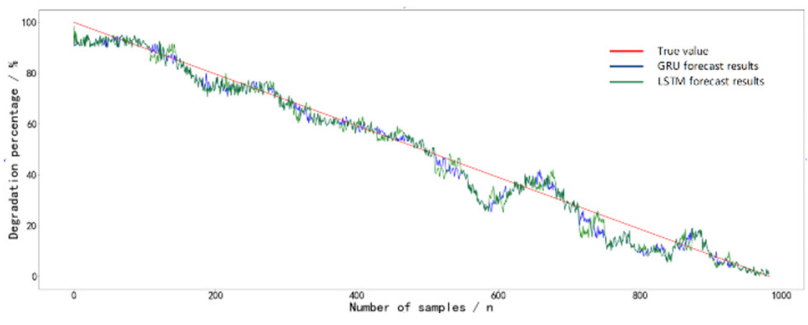

Fig.7. GRU and LSTM forecast results

Although the prediction results of Gru and LSTM models are basically the same, with the increase of the complexity of the models, the gap between them becomes larger. Gru takes 73 seconds in this prediction, while LSTM takes 98 seconds. Therefore, Gru network model can not only accurately predict the remaining service life of fan spindle bearing, but also improve the prediction efficiency of the model.

\section{Conclusion}

In the process of fan operation, the bearing vibration data is increasing, a large number of data makes the prediction model prone to redundant calculation and gradient disappear. In view of this phenomenon, a bearing life prediction method based on GRU network is proposed, and its effectiveness in the fan spindle bearing life prediction is verified. Compared with the prediction model of LSTM network, the prediction model proposed in this paper has obvious advantages in the efficiency of processing large amounts of data. In the future, we can find more effective methods to preprocess the data and optimize the model input to further improve the accuracy of the remaining service life of the fan spindle bearing.

\section{Reference}

1. Rh A, Lx A, Xl B, et al. Residual life predictions for ball bearings based on self-organizing map and back propagation neural network methods[J]. Mechanical Systems and Signal Processing, 2007, 21 (1):193-207.

2. Sun Lei, Jia Yunxian, Cai Liying, et al. Application of particle filter parameter estimation method in prediction of residual life of gearbox [J]. Vibration and shock, 2013, 32 (6): 6-12

3. Liu, Yang Junbin, Zhang Qing, et al. SOC prediction of lithium battery based on QPSO BP neural network $[\mathrm{J}]$. Journal of electronic measurement and instruments, 2013 (03): 44-48

4. Kozanecka D, Kozanecki Z, Tkacz E, et al. Experimental research of oil-free support systems to predict the high-speed rotor bearing dynamics[J]. International Journal of Dynamics and Control, 2014, 3(1):9-16. 